This is an author produced version of a paper published in Molecular Immunology.

This paper has been peer-reviewed and is proof-corrected, but does not include the journal pagination.

Citation for the published paper:

Waern, Ida; Jia, Juan; Pejler, Gunnar; Zcharia, Eyal; Vlodavsky, Israel; Li, Jin-Ping; Wernersson, Sara. (2010) Accumulation of Ym1 and formation of intracellular crystalline bodies inalveolar macrophages lacking heparanase. Molecular Immunology. Volume: 47, Number: 7-8, pp 1467-1475. http://dx.doi.org/10.1016/j.molimm.2010.02.004.

Access to the published version may require journal subscription. Published with permission from: Elsevier.

Epsilon Open Archive http://epsilon.slu.se 


\section{Accumulation of Ym1 and formation of intracellular crystalline bodies in alveolar macrophages lacking heparanase}

Ida Waern ${ }^{\mathrm{a}}$, Juan Jia ${ }^{\mathrm{b}}$, Gunnar Pejler ${ }^{\mathrm{a}}$, Eyal Zcharia ${ }^{\mathrm{c}}$, Israel Vlodavsky ${ }^{\mathrm{c}}$, Jin-Ping

$\mathrm{Li}^{\mathrm{b}}$, Sara Wernersson ${ }^{\mathrm{a},}{ }^{*}$

${ }^{a}$ Department of Anatomy, Physiology and Biochemistry, Swedish University of Agricultural Sciences, Sweden

${ }^{\mathrm{b}}$ Department of Medical Biochemistry and Microbiology, Uppsala University, Sweden

${ }^{\mathrm{c}}$ Cancer and Vascular Biology Research Center, The Bruce Rappaport Faculty of Medicine, Technion, Haifa 31096, Israel

${ }^{*}$ Corresponding author: Sara Wernersson, Swedish University of Agricultural Sciences, Department of Anatomy, Physiology and Biochemistry, BMC, Box 575, 75123 Uppsala, Sweden; E-mail: Sara.Wernersson@afb.slu.se; phone: +46-184714119; Fax: +46-18-550762 


\section{ABSTRACT}

Heparanase is a heparan sulfate (HS) degrading endoglucuronidase that has been implicated in cell migration and inflammatory conditions. Here we used mice deficient of heparanase $\left(\mathrm{Hpse}^{-/-}\right)$to study the impact of heparanase on airway leukocytes. Normal numbers of macrophages and lymphocytes were present in bronchoalveolar lavage fluid of Hpse-/- mice, indicating that heparanase is not essential for proper homing of leukocytes to airways. While lymphocytes from $\mathrm{Hpse}^{-/-}$mice displayed normal morphology, $\mathrm{Hpse}^{-/-}$alveolar macrophages showed a striking, age-dependent appearance of granule-like structures in the cytoplasm. Transmission electron microscopy revealed that these structures corresponded to membrane-enclosed crystalline bodies that closely resembled the intracellular crystals known to be formed by the HS-binding protein Ym1, suggesting that heparanase deficiency is associated with intracellular Ym1 deposition. Indeed, applying immunocytochemistry, we found markedly higher levels of intracellular Ym1 protein in $H p s e^{-/-}$versus $W T$ alveolar macrophages, and there was a significant correlation between levels of $\mathrm{Ym} 1$ protein detected by immunoblotting and amounts of crystalline material in BAL cells. Biosynthetic radio-labeling of the macrophages revealed accumulation of non-degraded HS chains in $H p s e^{-/-}$ alveolar macrophages. Together, these findings implicate heparanase in normal processing of HS in macrophages, and indicate that heparanase regulates intracellular Ym1 accumulation and crystal formation in airways.

Keywords: heparanase knockout mice; heparan sulfate; chitinase-like protein; alveolar macrophages; airway inflammation 
Abbreviations: BAL, bronchoalveolar lavage; BMMØ, bone marrow-derived macrophage; DTH, delayed type hypersensitivity; ECM, extracellular matrix; GAG, glycosaminoglycan; Hpse, heparanase; HS, heparan sulfate; HSPG, heparan sulfate proteoglycans; TEM, transmission electron microscopy. 


\section{Introduction}

Heparan sulfate proteoglycans (HSPGs) consist of protein cores to which sulfated and thereby negatively charged glycosaminoglycans (GAGs) of heparan sulfate (HS) type are attached. HSPGs are ubiquitously expressed, being present on the cell surface of most cell types as well as being one of the main components of the extracellular matrix (ECM). Cell surface HSPGs, e.g. of the syndecan or glypican families (Alexopoulou et al., 2007; Filmus et al., 2008), mediate cell-cell and cellmatrix contacts and function as co-receptors for various growth factors (Mythreye and Blobe, 2009). In addition, cell surface HSPGs lining the vascular endothelium act as sites for leukocyte attachment, thereby facilitating migration of blood-borne cells into tissues (Wang et al., 2005).

Heparanase is an endoglucuronidase that cleaves HS chains (Pikas et al., 1998). Heparanase may thus modify the structure of preformed HSPGs and hence regulate the plethora of processes in which HSPGs are implicated. Indeed, previous studies have suggested a role for heparanase in various pathophysiological conditions, including tumor metastasis (Escobar Galvis et al., 2007; Vlodavsky and Friedmann, 2001), experimental autoimmune disease (Lider et al., 1989), amyloidosis ( $\mathrm{Li}$ et al., 2005) and delayed type hypersensitivity (DTH) reactions (Edovitsky et al., 2006). A role for heparanase in tumour metastasis is supported by the strong correlation between levels of heparanase expression and metastatic potential of B16 melanoma (Vlodavsky et al., 1999) and T lymphoma (Goldshmidt et al., 2002b) cells. Further, it has been shown by RNA interference that suppression of heparanase expression ameliorates DTH reactions (Edovitsky et al., 
2006) as well as reduces lung colonization of B16 melanoma cells (Edovitsky et al., 2004). The role of heparanase in normal cell and tissue physiology is however not well characterized, although a role in tissue remodeling, vascularization and hair growth has been suggested applying transgenic mice overexpressing heparanase (Zcharia et al., 2004).

It is important to point out that most of the functional implications for heparanase have been derived from correlative or in vitro observations, and that detailed investigations of the in vivo function of heparanase have been hampered by the lack of relevant tools. The recent generation of a mouse strain lacking heparanase expression has opened new possibilities for determining its in vivo function (Zcharia et al., 2009). Heparanase deficient $\left(\mathrm{Hpse}^{-/-}\right)$mice do not demonstrate any major abnormalities under normal conditions, possibly due to an increased expression of matrix metalloproteases, compensating for the loss of heparanasemediated ECM degradation (Zcharia et al., 2009). Moreover, heparanase deficiency results in accumulation of non-degraded HS chains in different tissues.

Heparanase is highly expressed by numerous types of tumor cells, and by various cells of the immune system (Vlodavsky et al., 1992), including mast cells (Gong et al., 2003), eosinophils (Temkin et al., 2004), dendritic cells (Benhamron et al., 2006) and monocytes/macrophages (Sasaki et al., 2004). However, the role of heparanase in the context of immune cell function is only partially understood. Here we addressed this issue by studying the impact of heparanase deficiency on various leukocyte populations present in airways. We show that the absence of heparanase does not affect homing of lymphocytes or macrophages into the 
airways, nor does it affect lymphocyte morphology. However, lack of heparanase caused an accumulation of Ym1, a chitinase-like protein, accompanied by a striking appearance of intracellular crystalline bodies in alveolar macrophages. Moreover, the HS chains in heparanase mutant macrophages were longer than in WT macrophages. Together, these findings implicate heparanase in the regulation of macrophage homeostasis. 


\section{Materials and Methods}

\subsection{Mice and collection of bronchoalveolar lavage}

Heparanase-deficient $\left(\mathrm{Hpse}^{--}\right)$mice were generated as described (Zcharia et al., 2009) and were bred and maintained in the animal facility at Uppsala Biomedical Centre. Experimental groups were age and sex matched and $H p s e^{+/+}$littermates (WT mice) were used as controls. All animal experiments were approved by the local ethical committee. Bronchoalveolar lavage (BAL) fluid was collected by rinsing the lungs twice with $1 \mathrm{ml}$ HBSS. Total numbers of cells were counted and cytospin slides were prepared. In separate experiments the remaining cells were centrifuged and prepared for TEM or immunoblot analysis, and the cell-free BALsupernatants were stored in $-20^{\circ}$ until used.

\subsection{Transmission electron microscopy (TEM)}

BAL cells from 7 or 17 months old $H p s e^{-/-}$and $W T$ mice were pooled from 2 mice per group and prepared for TEM as described (Braga et al., 2007). Electron micrographs were generated using a Hitachi electron microscope (Hitachi Ltd., Tokyo, Japan) (Lukinius et al., 1995).

\subsection{Differential cell count and morphology}

Cytospin slides were stained with May-Grünwald/Giemsa (Merck, Darmstadt, Germany) and the numbers of different airway cells were determined by differential counting of at least 200 cells per slide. The altered morphology of alveolar macrophages, i.e. the presence of granules in the cytoplasm, was quantified by blinded scoring of at least 200 cells per slide. Cells with visual 
granules were given scores (1-3) corresponding to the degree of granulation and no score (0) was given for cells lacking granules. An average score per counted cell was determined. To evaluate the presence of highly sulfated GAGs, cells were stained with Toluidine blue or Alcian blue as described (Braga et al., 2007; Reimer et al., 2006).

\subsection{Immunocytochemistry}

For immunocytochemistry, cytospin slides were fixed in ice-cold acetone for 5 minutes. Cells were permeabilized and blocked by incubation for 30 minutes with PBS containing $0.1 \%$ saponin, $0.5 \%$ BSA, and $10 \%$ goat serum. Endogenous peroxidase was blocked with PBS containing $0.3 \% \mathrm{H}_{2} \mathrm{O}_{2}$ and $0.3 \%$ goat serum for 5 minutes. Cells were stained for $1 \mathrm{hr}$ with anti-Yml antibodies (StemCell Technologies, Vancouver, Canada) or anti-mouse $\operatorname{IgG}(\mathrm{H}+\mathrm{L})$ antibodies (Chemicon international, Temecula, CA, USA) diluted 1:200 in a blocking buffer (PBS with $0.1 \%$ saponin, $0.5 \%$ BSA, $2 \%$ goat serum, and $0.42 \mathrm{mM} \mathrm{NaCl}$ ). Rabbit IgG (Vector Laboratories, Burlingame, CA, USA) was used as a negative control. Cells were then incubated for $1 \mathrm{hr}$ with biotinylated goat anti-rabbit IgG diluted 1:200 in blocking buffer. The Vectastain ${ }^{\circledR}$ Elite $A B C$ reagent and DAB substrate kit for peroxidase were used for development (Vector Laboratories). Slides were counterstained for 2 minutes with Mayer's hematoxylin solution (Sigma-Aldrich, St. Louis, MO, USA) and mounted with VectaMount (Vector Laboratories).

\subsection{Generation of bone marrow derived macrophages}

To generate conditioned medium for macrophage differentiation, we cultured the colony stimulating factor (CSF)-1 secreting cell line L929 (provided by Dr Ulrich 
Blank, Inserm U699, Paris, France) in DMEM supplemented with 5\% FCS, 50 $\mu \mathrm{g} / \mathrm{ml}$ penicillin, $60 \mu \mathrm{g} / \mathrm{ml}$ streptomycin and $2 \mathrm{mM}$ L-glutamine (SVA, Uppsala, Sweden). Culture densities were initially $3 \times 10^{5}$ cells $/ \mathrm{ml}$, and after 8 days of culture, supernatants were collected, filtered and stored at $-20^{\circ} \mathrm{C}$ until used. Bone marrow cells from eight months old $W T$ or $H p s e^{-/-}$mice were collected from femura and tibia by flushing the bones with $2.5 \mathrm{ml}$ PBS. Red blood cells were removed by incubating cells for 2 minutes in $200 \mu 1$ lysis buffer $\left(0.15 \mathrm{M} \mathrm{NH}_{4} \mathrm{Cl}\right.$, 0.1mM EDTA, pH7.4). Cells were cultured overnight in complete medium (DMEM supplemented with 15\% FCS, 10\% L929-conditioned medium, $50 \mu \mathrm{g} / \mathrm{ml}$ penicillin, $60 \mu \mathrm{g} / \mathrm{ml}$ streptomycin and $2 \mathrm{mM}$ L-glutamine). Non-adherent cells, including monocyte precursors, were collected and $3 \times 10^{5}$ cells $/ \mathrm{ml}$ were added to Petri dishes and cultured for 10 days in complete medium before used in further analyses.

\subsection{FACS analysis}

To confirm the purity of bone marrow derived macrophages, flow cytometry was used to identify presence of cells expressing the macrophage marker CD11b. Cells were washed twice in PBS and diluted in flow cytometry buffer $(0.5 \% \mathrm{FCS} / \mathrm{PBS})$. Samples of $0.2 \times 10^{6}$ cells were stained for 30 minutes on ice with $0.5 \mu \mathrm{g}$ PElabeled anti-CD11b antibodies or with $0.5 \mu \mathrm{g}$ PE-labeled isotype controls (ImmunoTools, Friesoythe, Germany). After washing two times, cells were analyzed using a FACScan ${ }^{\circledR}$ flow cytometer and the CELLQuest ${ }^{\mathrm{TM}} 3.3$ software (Becton Dickinson, San Jose, CA, USA).

\subsection{Biosynthetic labeling and analysis of $H S$}


Bone marrow derived macrophages from $\mathrm{WT}$ or $\mathrm{Hpse}^{-/-}$mice were cultured at $0.56 \times 10^{6}$ cells $/ \mathrm{ml}$ in sulfate free medium supplemented with $2 \mathrm{mM} \mathrm{L}$-glutamine, $1 \%$ pest, 10\% L929-conditioned medium, 10\% FCS (dialyzed against PBS), and $100 \mu \mathrm{Ci} / \mathrm{ml} \mathrm{Na}^{35} \mathrm{SO}_{4}$ (specific activity $1,500 \mathrm{Ci} / \mathrm{mmol}$; PerkinElmer, Waltham, MA, USA). After 24 hours, HS was isolated from medium or cell lysates as described (Escobar Galvis et al., 2007). The purified ${ }^{35}$ S-labeled HS was analyzed on a superose-6 column (GE Healthcare Biosciences, Uppsala, Sweden) in a buffer of $0.5 \mathrm{M} \mathrm{NH}_{4} \mathrm{HCO}_{3}$.

\subsection{Immunoblot analysis}

Ten $\mu 1$ of BAL fluid or BAL cell pellets from 13-17 months old WT or Hpse ${ }^{-/-}$mice were separated under reducing conditions on $12 \%$ SDS-PAGE gels. Proteins were subsequently blotted onto polyvinylidene difluoride membranes, followed by incubation with LI-COR blocking buffer (LI-COR Biosciences, Cambridge, UK) for $1 \mathrm{~h}$ at room temperature. Membranes were stained o.n. at $4{ }^{\circ} \mathrm{C}$ with anti-Ym1 (StemCell Technologies) antibodies diluted 1:1000 in blocking buffer. After washing with $0.1 \%$ Tween 20 in TBS, the membrane was incubated with IR Dye 800-conjugated donkey anti-rabbit Ig (LI-COR Biosciences, dilution 1:1000) in blocking buffer. Visualization and densitometry analyses were performed using Odyssey IR imaging system (LI-COR Biosciences). Data were measured as counts $/ \mathrm{mm}^{2}$ and normalized to the loading control, i.e. results are expressed as the ratio between sample value for Ym1 and the corresponding value for $\beta$-actin.

\subsection{Statistical analyses}


Statistical differences between $W T$ and $H p s e^{-/-}$mice were determined by MannWhitney U test, and correlations were analyzed by Spearman test, using the GraphPad Prism 4.0 (GraphPad Software, Inc, San Diego, CA, USA). 


\section{Results}

\subsection{Normal homing of leukocytes to airways in the absence of heparanase}

Heparanase has previously been implicated in inflammatory processes and cell migration (Benhamron et al., 2006; Edovitsky et al., 2006; Sasaki et al., 2004), although the precise impact of heparanase on leukocyte populations in vivo has not been evaluated. To address this issue, we examined the effect of heparanase deficiency on leukocytes present in bronhcoalveolar lavage (BAL) fluid. As shown in Fig 1A, the BAL cell populations from both $W T$ and $H p s e^{-/-}$mice were mainly composed of macrophages. There were very few lymphocytes (1.3\% in average) and almost no neutrophils or eosinophils in BAL, indicating that there was no spontaneous ongoing inflammatory reaction in the airways. There was a tendency for higher numbers of macrophages and lymphocytes in the Hpse $e^{-/}$mice, but the difference between $W T$ and $H p s e^{-/-}$mice did not reach statistical significance (Fig. 1A). These results demonstrate that, under normal conditions, heparanase has no major impact on migration of macrophages and lymphocytes to the airway lumen.

\subsection{Granule-like structures in Hpse-- alveolar macrophages}

Examination of the morphology of May-Grünwald/Giemsa-stained BAL leukocyte populations showed that Hpse $e^{-/}$lymphocytes did not differ morphologically from the corresponding WT cells (not shown). In contrast, Hpse $e^{-/}$macrophages showed a striking presence of purple-stained intracellular, granule-like structures, which was not seen in $W T$ macrophages (Fig. 1B). A systematic scoring for the presence of granule-like structures in alveolar macrophages from 3-17 months old WT and $\mathrm{Hpse}^{-/-}$mice showed that the extent of granulation correlated strongly with the age 
of animals, with a more pronounced granulation seen in old (i.e. 7 and 13-17 months) vs. young (i.e. 3 months) mice (Fig. 1B and C). Although some signs of granulation were also seen in alveolar macrophages from old WT mice, the extent and intensity of granulation was markedly higher in corresponding macrophages derived from $\mathrm{Hpse}^{-/-}$mice (Fig. 1B and C). Notably, granule-like structures were not seen in peritoneal macrophages from $\mathrm{WT}$ or $\mathrm{Hpse}^{-/-}$mice (not shown), suggesting that this phenotype was restricted to the airways.

Potentially, the increased granulation of alveolar macrophages might be associated with accumulation of cellular HS due to the absence of heparanase. To address this possibility, we stained for sulfated GAGs using Alcian blue and Toluidine blue (Fig. 2). Compared with peritoneal macrophages, alveolar macrophages stained more intensely with Alcian blue (Fig. 2A), suggesting a higher level of cellassociated GAGs in airways as compared to peritoneal macrophages. However, there was no significant difference in the staining intensity between $W T$ and $H p s e^{-/-}$ macrophages. Moreover, alveolar macrophages did not demonstrate metachromatic staining with Toluidine blue (Fig. 2B), indicating that there was no substantial accumulation of highly sulfated GAGs in these cells.

\subsection{Needle-shaped crystalline bodies in Hpse $e^{-/}$alveolar macrophages}

To further examine the nature of the granule-like structures present in $H p s e^{-/-}$ macrophages, alveolar macrophages were examined by transmission electron microscopy (TEM). The TEM analysis revealed a striking morphology of electron dense and needle-shaped crystalline bodies in alveolar macrophages derived from $H_{p s e^{-/}}$animals, structures that were totally absent in corresponding WT cells (Fig. 
3). The crystalline bodies were surrounded by membranes, indicating that they had developed within membrane-enclosed organelles such as lysosomes, ER or Golgi.

\subsection{Accumulation of Ym1 in alveolar macrophages from Hpse-- mice}

Next, we sought to identify the macrophage-associated crystalline material. Alveolar macrophages constitutively express Ym1, a chitinase-like protein that previously has been demonstrated to exhibit a marked tendency to spontaneously form crystals in vitro (Chang et al., 2001). Moreover, Ym1 is known to form needle-shaped intracellular crystals (Harbord et al., 2002; Nio et al., 2004) that closely resemble the crystals found in $H p s e^{-/}$alveolar macrophages (Fig. 3). Hence, one plausible scenario is that the crystalline bodies seen in $H p s e^{-/-}$alveolar macrophages contain Ym1. To address this possibility, we applied immunocytochemistry to stain for $\mathrm{Ym} 1$ in alveolar and peritoneal macrophages from $W T$ and $\mathrm{Hpse}^{-/}$mice. As shown in Fig. 4A and B, alveolar macrophages from both $W T$ and $H p s e^{-/}$mice stained positively for $\mathrm{Ym} 1$ whereas peritoneal macrophages were negative, in agreement with the reported absence of Ym1 in unstimulated peritoneal macrophages (Hung et al., 2002). However, Hpse $e^{-/}$ macrophages stained considerably stronger for $\mathrm{Ym} 1$ than did corresponding $W T$ cells, indicating that Hpse deficiency indeed results in accumulation of cellassociated Ym1. Noteworthy, the staining showed a patchy, intracellular appearance, compatible with the presence of Ym1 within membrane-enclosed organelles (Fig 4A).

Although Ym1 is a likely candidate to explain the altered morphology of $\mathrm{Hpse}^{-/-}$ alveolar macrophages, there are other known crystal-forming proteins to consider. 
Of particular interest are immunoglobulin (Ig) light and heavy chains, which are known to occasionally form needle-shaped intracellular crystals in tissue macrophages of patients with lymphoproliferative diseases (Lebeau et al., 2002). However, the alveolar macrophages stained weakly and non-patchy for IgG, with no differential extent of staining in $W T$ versus $H p s e^{-/-}$cells (Fig. 4C), suggesting that the crystalline bodies seen in Hpse $e^{-/}$macrophages do not originate from accumulated IgG.

To further substantiate the association between intracellular Ym1 and crystalline formation, we evaluated Ym1 protein levels in both the cell- and fluid- fraction of BAL from 13-17 months old mice, using immunoblot analysis. At this age there is a significant difference between $W T$ and $H p s e^{-/-}$mice in the visual appearance of intracellular crystalline material, although a low degree of granulation is also found in $W T$ mice (Fig 1C). As shown in figure 4D, high amounts of cell-associated Ym1 were apparent in three out of four samples collected from $\mathrm{Hpse}^{-/-}$mice, and only in one of three samples collected from WT mice. Notably, the Hpse $e^{-/}$sample with low levels of Ym1 protein was also given low scores for intracellular granulation (Fig 4E). In fact, this sample was given the lowest scores within the group of 13-17 months old Hpse-- mice (Fig 1C) and is therefore not a representative sample. When analyzing samples from both genotypes, there was a statistically significant correlation between intracellular Ym1 levels and the amount of crystalline material in BAL cells (Fig 4E). In contrast, extracellular Ym1 levels did not correlate with intracellular crystals (Fig 4F) and the levels of Yml in cell-free BAL fluid did not differ between $H p s e^{-/-}$and WT mice (Fig 4D). Collectively, these results strongly suggest that heparanase deficiency results in accumulation of $\mathrm{Ym} 1$ in airway 
macrophages and that $\mathrm{Ym} 1$ is the crystal-forming unit in $H p s e^{-/-}$alveolar macrophages.

\subsection{Non-degraded HS chains in bone marrow derived Hpse-- macrophages}

The results described above indicate that the absence of heparanase affects the homeostasis of alveolar macrophages. To study the possible involvement of macrophage HSPGs in this effect, biosynthetic labeling experiments were carried out. Because of the low number of macrophages that can be recovered from BAL fluid, the biosynthetic labeling experiments were instead performed using macrophages derived by in vitro differentiation of bone marrow precursors (bone marrow-derived macrophages; BMMØs). As shown in Fig. 5A, cells strongly positive for the macrophage marker $\mathrm{CD} 11 \mathrm{~b}$ were obtained by in vitro differentiation of bone marrow precursors in the presence of L929-conditioned medium. The numbers of CD11b-positive cells and the intensity of CD11b staining did not differ between $W T$ and Hpse $e^{-/}$cells, indicating that absence of heparanase does not influence macrophage differentiation (Fig 5A). Moreover, staining with May-Grünwald/Giemsa did not reveal any obvious morphological difference between BMMØs from $W T$ and $H s p e^{-/-}$mice (not shown). In contrast to the BAL macrophages, no signs of intracellular granulation were seen in $H p s e^{-/-}$BMMØs. However, as this phenotype appears to be age-dependent (see Fig. 1B and C), its absence in BMMØs after 10 days of culture is not unexpected.

Having confirmed the macrophage phenotype of BMMØs (Fig 5A), we metabolically labeled the cells with ${ }^{35} \mathrm{~S}$-sulfate, followed by purification of cellassociated and secreted HS. Gel filtration analysis showed that HS chains 
recovered from both the cell-associated and secreted HS isolated from $\mathrm{Hpse}^{-/-}$ BMMØs are longer than HS extracted from $W T$ BMMØs (Fig. 5B), indicating that lack of heparanase leads to accumulation of non-degraded HS chains in $\mathrm{Hpse}^{-/-}$ BMMØs. 


\section{Discussion}

In the present study we establish that heparanase decisively affects macrophage homeostasis. This was demonstrated by the findings that macrophages lacking heparanase contain non-degraded HS chains and that alveolar macrophages from $\mathrm{Hpse}^{-/-}$mice exhibit an age-dependent increase in granule-like structures, identified as electron dense crystalline bodies surrounded by intracellular membranes. Moreover, the altered morphology of Hpse-/- alveolar macrophages was associated with an increased patchy staining of intracellular Ym1, a protein known to form intracellular crystals (Nio et al., 2004).

Heparanase expression has been detected in a variety of leukocyte populations, including monocytes/macrophages, dendritic cells, neutrophils, $\mathrm{CD}^{+} \mathrm{T}$ cells, mast cells and eosinophils (Benhamron et al., 2006; Temkin et al., 2004; Vlodavsky et al., 1992). Interestingly, heparanase expressed on the surface of dendritic cells and macrophages has been shown to promote migration of these cells, by degrading ECM barriers (Benhamron et al., 2006; Sasaki et al., 2004). Moreover, heparanase can enhance cell adhesion independent of its enzymatic activity, and thereby contribute to cell motility (Goldshmidt et al., 2003; Sotnikov et al., 2004). These findings suggest a role for heparanase in regulating leukocyte migration processes. We therefore hypothesized that absence of heparanase may lead to defective leukocyte migration, possibly resulting in a reduced number of leukocytes in the BAL. We show, however, that the absence of heparanase does not influence these leukocyte populations adversely. Thus, it appears that under normal conditions heparanase expression is not essential for migration of leukocytes in to the 
airways. However, it remains to be elucidated whether heparanase influences migration of the corresponding cell types during an inflammatory reaction.

A striking and novel finding in this study was the appearance of granulated alveolar macrophages in aged mice lacking heparanase, as detected by May Grünwald/Giemsa staining. This phenomenon was exclusively seen in the BAL macrophage population, and was totally absent in other airway leukocytes and in peritoneal macrophages. A closer examination of the macrophages by TEM revealed that the granule-like structures had a distinct electron dense needle-shaped crystalline appearance. Moreover, the TEM analysis showed that the intracellular crystalline bodies were enclosed by membranes. One possible interpretation of these findings is that the crystalline bodies are located in lysosomes, and that they arise as a consequence of impaired regulation of compounds accumulating in the lysosome due to the absence of heparanase. In line with such a possibility, heparanase has previously been identified in intracellular compartments, including late endosomes and lysosomes (Goldshmidt et al., 2002a) in which proheparanase is processed and activated (Zetser et al., 2004). Moreover, heparanase activity is optimal at pH5-6 (Gilat et al., 1995), further supporting a physiological function of heparanase restricted to cellular compartments having acidic $\mathrm{pH}$, such as lysosomes.

An obvious possibility would be that the crystalline bodies in $H p s e^{-/-}$macrophages contain HSPGs that accumulate in lysosomes as a result of heparanase-deficiency. However, since the absence of heparanase did not result in a substantial accumulation of cell-associated HSPGs as judged by biosynthetic labeling 
experiments and by basic-dye cell staining, it appears less likely that HSPGs are predominant components of the crystals. Another possibility is that accumulation of crystalline bodies is linked to the marked increase in HS chain length shown to accompany the absence of heparanase. Although we cannot precisely explain how the increased HS chain length could account for the accumulation of crystalline material, one potential scenario could be that abnormally long HS chains may serve as a support that accumulates multiple crystal-forming molecules thus facilitating crystal formation. This would be in some analogy with the proposed contribution of HSPGs to amyloid formation (Stevens and Kisilevsky, 2000).

A crucial issue is to identify the crystal-forming unit in $H p s e^{-/-}$alveolar macrophages. Here we present several observations suggesting that the chitinaselike protein $\mathrm{Ym} 1$ is a main candidate. Most importantly, we demonstrate a statistically significant correlation between the extent of granule-like structures in alveolar macrophages and the amounts of intracellular Ym1 in the BAL cell fraction. Moreover, there was a markedly increased Ym1 immunostaining in Hpse ${ }^{\star}$ alveolar macrophages as compared to $W T$ counterparts, suggesting an intracellular accumulation of Ym1 protein in the absence of heparanase. Notably, staining of Ym1 was irregular with several intensely stained regions surrounded by non-stained areas, compatible with the presence of Ym1 within membranesurrounded organelles. Ym1 exhibits several known features that are in agreement with this notion. Firstly, Ym1 is known to easily form crystals in vitro (Chang et al., 2001) and intracellular crystalline bodies in vivo (Nio et al., 2004), in particular in alveolar macrophages from aged immunodeficient mice (Guo et al., 2000; Harbord et al., 2002). Secondly, since HS and heparin are physiological ligands to 
Ym1 (Chang et al., 2001), it may be speculated that changes in HS turnover affect Ym1 aggregation and crystallization. Finally, Ym1 is selectively expressed by alveolar macrophages and immature neutrophils under normal conditions (Nio et al., 2004) and is only expressed by peritoneal macrophages upon LPS-stimulation (Hung et al., 2002) or differentiation into alternatively activated macrophages (Kreider et al., 2007; Rauh et al., 2005). The selective expression of Ym1 in alveolar, but not peritoneal macrophages is well in line with the appearance of granule-like structures only in the former cell type. Collectively, these findings strongly suggest that $\mathrm{Ym} 1$ is the crystal-forming unit in Hpse ${ }^{-/-}$alveolar macrophages.

Ym1 belongs to a group of chitinase-like proteins, including Ym2 and BRP-39 in mice, and YKL-40 in humans (Bussink et al., 2007). Increased expression of these proteins has been associated with inflammatory diseases, in particular with allergic asthma (reviewed in (Lee et al., 2008; Shuhui et al., 2009). The physiological function of $\mathrm{Ym} 1$ is not clear, but a role in eosinophil chemotaxis (Owhashi et al., 2000) and promotion of cytokine production (Cai et al., 2009) has been suggested. Interestingly, $\mathrm{Ym} 1$ has been reported to exhibit a weak ability to cleave $N$ acetylglucosamine units (Guo et al., 2000; Harbord et al., 2002), suggesting that Ym1 may not only bind to HS but can possibly also cleave HS, and thus function in a manner similar to heparanase. However, the ability of Ym1 to process HS remains to be established.

In conclusion, the present study indicates that heparanase has a physiological role in airway cell homeostasis, by regulating the morphology of alveolar macrophages 
and by controlling the levels of intracellular Ym1. Considering the findings presented here and the implication of heparanase in inflammatory processes in general (Li and Vlodavsky, 2009), it may be anticipated that heparanase has a role in the regulation of airway inflammatory diseases. 


\section{Acknowledgement}

This work was supported by the Vårdal Foundation, the Swedish Society of Medicine, the Magnus Bergvall Foundation, the Tore Nilson Foundation, and the Åke Wiberg Foundation and Swedish Research Council, (K2009-67X-21128-013). We thank Agneta Lukinius for preparation and analysis of cells using transmission electron microscopy. 


\section{References}

Alexopoulou A. N., Multhaupt H. A. and Couchman J. R. (2007) Syndecans in wound healing, inflammation and vascular biology. Int J Biochem Cell Biol $39,505-28$.

Benhamron S., Nechushtan H., Verbovetski I., Krispin A., Abboud-Jarrous G., Zcharia E., Edovitsky E., Nahari E., Peretz T., Vlodavsky I. and Mevorach D. (2006) Translocation of active heparanase to cell surface regulates degradation of extracellular matrix heparan sulfate upon transmigration of mature monocyte-derived dendritic cells. J Immunol 176, 6417-24.

Braga T., Grujic M., Lukinius A., Hellman L., Åbrink M. and Pejler G. (2007) Serglycin proteoglycan is required for secretory granule integrity in mucosal mast cells. Biochem J 403, 49-57.

Bussink A. P., Speijer D., Aerts J. M. and Boot R. G. (2007) Evolution of mammalian chitinase(-like) members of family 18 glycosyl hydrolases. Genetics.

Cai Y., Kumar R. K., Zhou J., Foster P. S. and Webb D. C. (2009) Ym1/2 promotes Th2 cytokine expression by inhibiting 12/15(S)-lipoxygenase: identification of a novel pathway for regulating allergic inflammation. $J$ Immunol 182, 5393-9.

Chang N. C., Hung S. I., Hwa K. Y., Kato I., Chen J. E., Liu C. H. and Chang A. C. (2001) A macrophage protein, Ym1, transiently expressed during inflammation is a novel mammalian lectin. J Biol Chem 276, 17497-506. 
Edovitsky E., Elkin M., Zcharia E., Peretz T. and Vlodavsky I. (2004) Heparanase gene silencing, tumor invasiveness, angiogenesis, and metastasis. $J$ Natl Cancer Inst 96, 1219-30.

Edovitsky E., Lerner I., Zcharia E., Peretz T., Vlodavsky I. and Elkin M. (2006) Role of endothelial heparanase in delayed-type hypersensitivity. Blood 107, 3609-16.

Escobar Galvis M. L., Jia J., Zhang X., Jastrebova N., Spillmann D., Gottfridsson E., van Kuppevelt T. H., Zcharia E., Vlodavsky I., Lindahl U. and Li J. P. (2007) Transgenic or tumor-induced expression of heparanase upregulates sulfation of heparan sulfate. Nat Chem Biol 3, 773-8.

Filmus J., Capurro M. and Rast J. (2008) Glypicans. Genome Biol 9, 224.

Gilat D., Hershkoviz R., Goldkorn I., Cahalon L., Korner G., Vlodavsky I. and Lider O. (1995) Molecular behavior adapts to context: heparanase functions as an extracellular matrix-degrading enzyme or as a $\mathrm{T}$ cell adhesion molecule, depending on the local pH. J Exp Med 181, 1929-34.

Goldshmidt O., Nadav L., Aingorn H., Irit C., Feinstein N., Ilan N., Zamir E., Geiger B., Vlodavsky I. and Katz B. Z. (2002a) Human heparanase is localized within lysosomes in a stable form. Exp Cell Res 281, 50-62.

Goldshmidt O., Zcharia E., Abramovitch R., Metzger S., Aingorn H., Friedmann Y., Schirrmacher V., Mitrani E. and Vlodavsky I. (2002b) Cell surface expression and secretion of heparanase markedly promote tumor angiogenesis and metastasis. Proc Natl Acad Sci US A 99, 10031-6.

Goldshmidt O., Zcharia E., Cohen M., Aingorn H., Cohen I., Nadav L., Katz B. Z., Geiger B. and Vlodavsky I. (2003) Heparanase mediates cell adhesion independent of its enzymatic activity. Faseb $J$ 17, 1015-25. 
Gong F., Jemth P., Escobar Galvis M. L., Vlodavsky I., Horner A., Lindahl U. and Li J. P. (2003) Processing of macromolecular heparin by heparanase. J Biol Chem 278, 35152-8.

Guo L., Johnson R. S. and Schuh J. C. (2000) Biochemical characterization of endogenously formed eosinophilic crystals in the lungs of mice. $J$ Biol Chem 275, 8032-7.

Harbord M., Novelli M., Canas B., Power D., Davis C., Godovac-Zimmermann J., Roes J. and Segal A. W. (2002) Ym1 is a neutrophil granule protein that crystallizes in p47phox-deficient mice. J Biol Chem 277, 5468-75.

Hung S. I., Chang A. C., Kato I. and Chang N. C. (2002) Transient expression of Ym1, a heparin-binding lectin, during developmental hematopoiesis and inflammation. J Leukoc Biol 72, 72-82.

Kreider T., Anthony R. M., Urban J. F., Jr. and Gause W. C. (2007) Alternatively activated macrophages in helminth infections. Curr Opin Immunol 19, 44853.

Lebeau A., Zeindl-Eberhart E., Muller E. C., Muller-Hocker J., Jungblut P. R., Emmerich B. and Lohrs U. (2002) Generalized crystal-storing histiocytosis associated with monoclonal gammopathy: molecular analysis of a disorder with rapid clinical course and review of the literature. Blood 100, 1817-27.

Lee C. G., Da Silva C. A., Lee J. Y., Hartl D. and Elias J. A. (2008) Chitin regulation of immune responses: an old molecule with new roles. Curr Opin Immunol 20, 684-9.

Li J. P., Galvis M. L., Gong F., Zhang X., Zcharia E., Metzger S., Vlodavsky I., Kisilevsky R. and Lindahl U. (2005) In vivo fragmentation of heparan 
sulfate by heparanase overexpression renders mice resistant to amyloid protein A amyloidosis. Proc Natl Acad Sci U S A 102, 6473-7.

Li J. P. and Vlodavsky I. (2009) Heparin, heparan sulfate and heparanase in inflammatory reactions. Thromb Haemost 102, 823-8.

Lider O., Baharav E., Mekori Y. A., Miller T., Naparstek Y., Vlodavsky I. and Cohen I. R. (1989) Suppression of experimental autoimmune diseases and prolongation of allograft survival by treatment of animals with low doses of heparins. J Clin Invest 83, 752-6.

Lukinius A., Jansson L. and Korsgren O. (1995) Ultrastructural evidence for blood microvessels devoid of an endothelial cell lining in transplanted pancreatic islets. Am J Pathol 146, 429-35.

Mythreye K. and Blobe G. C. (2009) Proteoglycan signaling co-receptors: roles in cell adhesion, migration and invasion. Cell Signal 21, 1548-58.

Nio J., Fujimoto W., Konno A., Kon Y., Owhashi M. and Iwanaga T. (2004) Cellular expression of murine Ym1 and Ym2, chitinase family proteins, as revealed by in situ hybridization and immunohistochemistry. Histochem Cell Biol 121, 473-82.

Owhashi M., Arita H. and Hayai N. (2000) Identification of a novel eosinophil chemotactic cytokine (ECF-L) as a chitinase family protein. J Biol Chem $275,1279-86$.

Pikas D. S., Li J. P., Vlodavsky I. and Lindahl U. (1998) Substrate specificity of heparanases from human hepatoma and platelets. $J$ Biol Chem 273, 187707. 
Rauh M. J., Ho V., Pereira C., Sham A., Sly L. M., Lam V., Huxham L., Minchinton A. I., Mui A. and Krystal G. (2005) SHIP represses the generation of alternatively activated macrophages. Immunity 23, 361-74.

Reimer J. M., Magnusson S., Juremalm M., Nilsson G., Hellman L. and Wernersson S. (2006) Isolation of transcriptionally active umbilical cord blood-derived basophils expressing FcepsilonRI, HLA-DR and CD203c. Allergy 61, 1063-70.

Sasaki N., Higashi N., Taka T., Nakajima M. and Irimura T. (2004) Cell surface localization of heparanase on macrophages regulates degradation of extracellular matrix heparan sulfate. J Immunol 172, 3830-5.

Shuhui L., Mok Y. K. and Wong W. S. (2009) Role of mammalian chitinases in asthma. Int Arch Allergy Immunol 149, 369-77.

Sotnikov I., Hershkoviz R., Grabovsky V., Ilan N., Cahalon L., Vlodavsky I., Alon R. and Lider O. (2004) Enzymatically quiescent heparanase augments T cell interactions with VCAM-1 and extracellular matrix components under versatile dynamic contexts. $J$ Immunol 172, 5185-93.

Stevens F. J. and Kisilevsky R. (2000) Immunoglobulin light chains, glycosaminoglycans, and amyloid. Cell Mol Life Sci 57, 441-9.

Temkin V., Aingorn H., Puxeddu I., Goldshmidt O., Zcharia E., Gleich G. J., Vlodavsky I. and Levi-Schaffer F. (2004) Eosinophil major basic protein: first identified natural heparanase-inhibiting protein. $J$ Allergy Clin Immunol 113, 703-9.

Wang L., Fuster M., Sriramarao P. and Esko J. D. (2005) Endothelial heparan sulfate deficiency impairs L-selectin- and chemokine-mediated neutrophil trafficking during inflammatory responses. Nat Immunol 6, 902-10. 
Vlodavsky I., Eldor A., Haimovitz-Friedman A., Matzner Y., Ishai-Michaeli R., Lider O., Naparstek Y., Cohen I. R. and Fuks Z. (1992) Expression of heparanase by platelets and circulating cells of the immune system: possible involvement in diapedesis and extravasation. Invasion Metastasis $12,112-27$.

Vlodavsky I. and Friedmann Y. (2001) Molecular properties and involvement of heparanase in cancer metastasis and angiogenesis. J Clin Invest 108, 341-7.

Vlodavsky I., Friedmann Y., Elkin M., Aingorn H., Atzmon R., Ishai-Michaeli R., Bitan M., Pappo O., Peretz T., Michal I., Spector L. and Pecker I. (1999) Mammalian heparanase: gene cloning, expression and function in tumor progression and metastasis. Nat Med 5, 793-802.

Zcharia E., Jia J., Zhang X., Baraz L., Lindahl U., Peretz T., Vlodavsky I. and Li J. P. (2009) Newly generated heparanase knock-out mice unravel coregulation of heparanase and matrix metalloproteinases. PLoS One 4, e5181.

Zcharia E., Metzger S., Chajek-Shaul T., Aingorn H., Elkin M., Friedmann Y., Weinstein T., Li J. P., Lindahl U. and Vlodavsky I. (2004) Transgenic expression of mammalian heparanase uncovers physiological functions of heparan sulfate in tissue morphogenesis, vascularization, and feeding behavior. Faseb $J$ 18, 252-63.

Zetser A., Levy-Adam F., Kaplan V., Gingis-Velitski S., Bashenko Y., Schubert S., Flugelman M. Y., Vlodavsky I. and Ilan N. (2004) Processing and activation of latent heparanase occurs in lysosomes. J Cell Sci 117, 224958. 


\section{Figure captions}

Fig. 1. Effect of heparanase deficiency on airway leukocyte composition and morphology. Bronchoalveolar lavage (BAL) cells were collected from $H p s e^{-/-}$and WT mice of different ages and stained with May-Grünwald/Giemsa. (A)

Differential counts of BAL leukocytes from 7 months old mice $(\mathrm{n}=11$; pooled from 3 independent experiments): tot, total cells; mac, macrophages; lym, lymphocytes; neu, neutrophils; eos, eosinophils. The graph shows the mean total number of cells \pm SEM. (B) Representative images showing the presence of granule-like material in $\mathrm{Hpse}^{-/-}$alveolar macrophages from $3(\mathrm{n}=3), 7(\mathrm{n}=11$; pooled from 3 independent experiments) and 13-17 ( $\mathrm{n}=6$; pooled from 2 independent experiments) months old mice. The extent of granulation was quantified by blinded scoring and the results are presented as mean scores \pm SEM $(C)$. Statistical differences were determined by Mann-Whitney U test: ***, $P<0.001{ }^{*}, P<0.05$; not significant (ns), $P>$ 0.05 .

Fig 2. Basic dye staining of $H p s e^{-/-}$and $W T$ macrophages. Alveolar macrophages (AM) and peritoneal macrophages $(P M)$ from 7 months old mice $(n=3-4)$ were stained with Alcian blue (A) or Toluidine blue (B) dye. Stained peritoneal mast cells (MC) are shown as positive controls. Representative images are shown. ND, not done. 400x original magnification.

Fig 3. Transmission electron microscopy (TEM) analysis of alveolar macrophages. Upper panels: Representative micrographs showing the morphology of WT and $\mathrm{Hpse}^{-/-}$macrophages. Lower panels: Higher magnification of selected areas marked 
by squares in the upper panels. Note the abundance of electron dense crystalline material surrounded by membranes (arrows) in the $\mathrm{Hpse}^{-/-}$macrophage. $\mathrm{N}$, nucleus; L, lysosome. Original magnification x 9,000.

Fig. 4. Heparanase deficiency results in increased levels of Ym1 in alveolar macrophages. BAL and peritoneal cells were collected from 7 months old $W T$ and $H_{p s e} e^{-/}$mice (A-C). Cytospin preparations of BAL cells (A, C) and peritoneal cells (B) were stained with anti-Ym1 (A, B), anti-IgG heavy and light chains (C), or isotype specific control antibodies. Images are representatives of 6 (A) or 2 (B and C) independent experiments. BAL cells and cell-free BAL fluid were collected from 13-17 months old mice and subjected to SDS-PAGE followed by immunoblotting with anti-Yml antibodies (D). Band intensities (counts $/ \mathrm{mm}^{2}$ ) were measured and the results are expressed as normalized densitometry values, i.e. ratios between samples and the corresponding $\beta$-actin loading control (E, F). Amounts of intracellular granule-like structures were analyzed by blinded scoring of May-Grünwald/Giemsa-stained cells and were plotted against corresponding densitometry values (E, F). The degree of granulation was significantly associated with intracellular Ym1 levels (E) but not with extracellular Ym1 levels (F) in BAL, as determined by Spearman correlation test $(P<0.05)$.

Fig. 5. Effects of heparanase deficiency on the molecular size of HS isolated from bone marrow-derived macrophages (BMMØs). (A) Flow cytometry analysis demonstrating that $W T$ and $H p s e^{-/-}$BMMØs show equal expression of the macrophage marker CD11b. (B) Gel chromatography on Superose 6 was applied 
to analyze biosynthetically ${ }^{35} \mathrm{~S}$-sulfate-labeled secreted (upper panel) and cellassociated (lower panel) HS from $W T$ and $H p s e^{-/-}$BMMØ. 
Fig.1
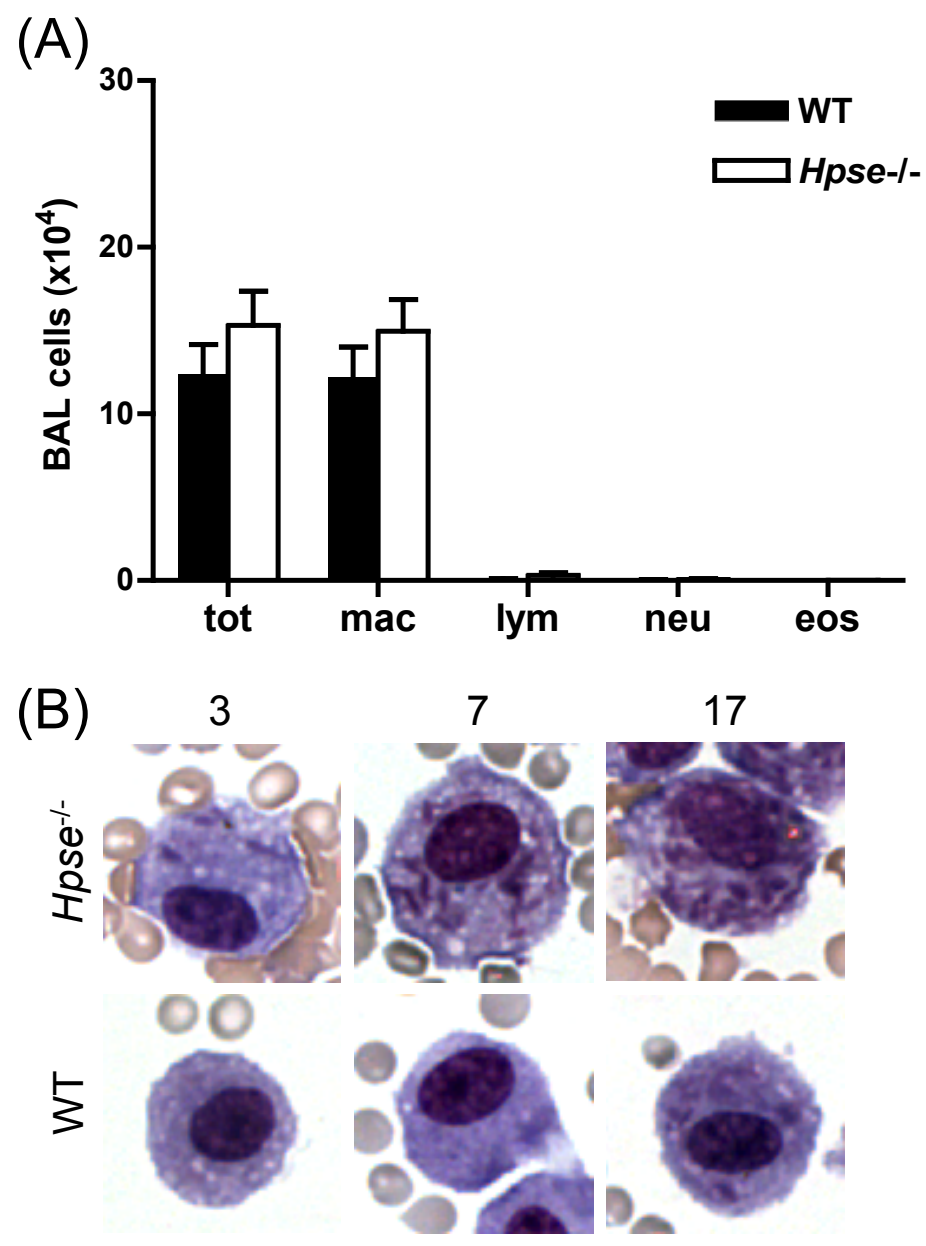

(C)

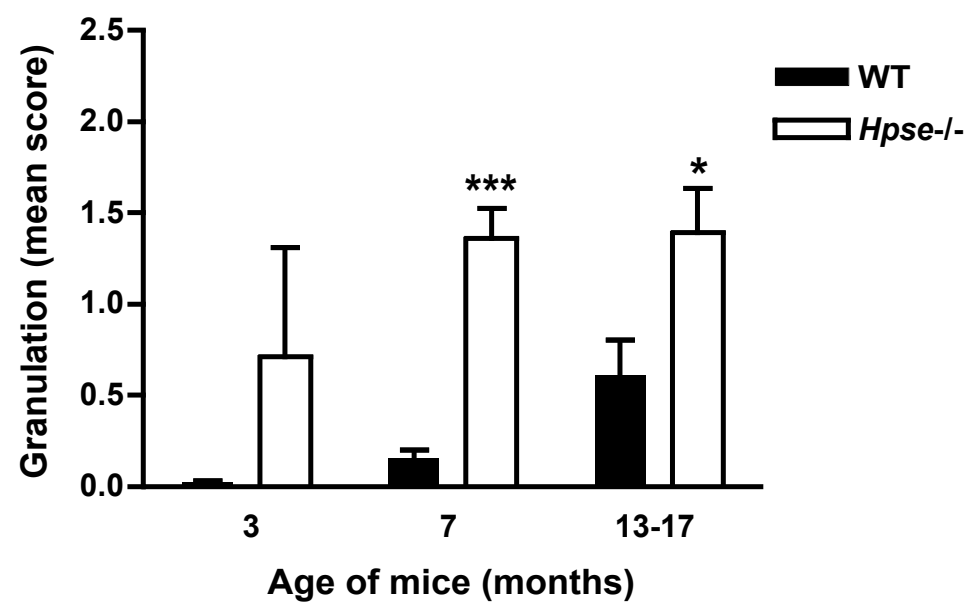


Fig.2

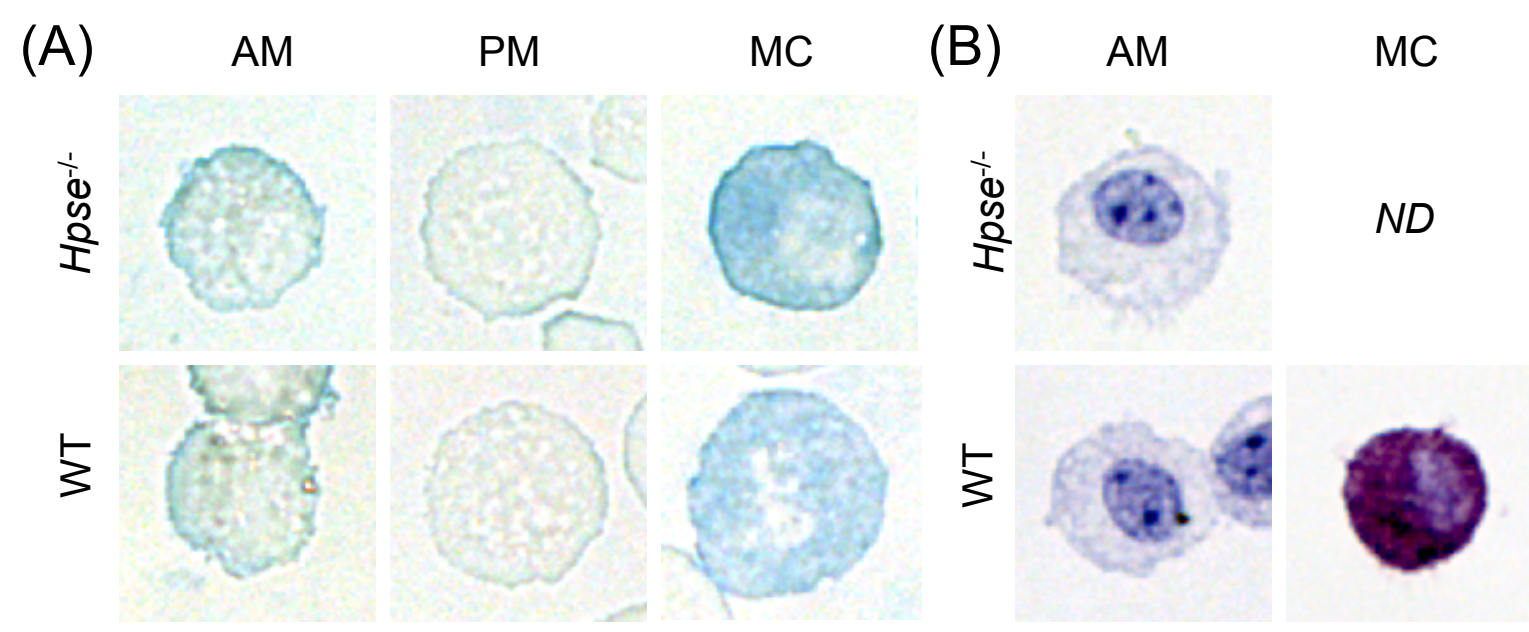


Fig.3
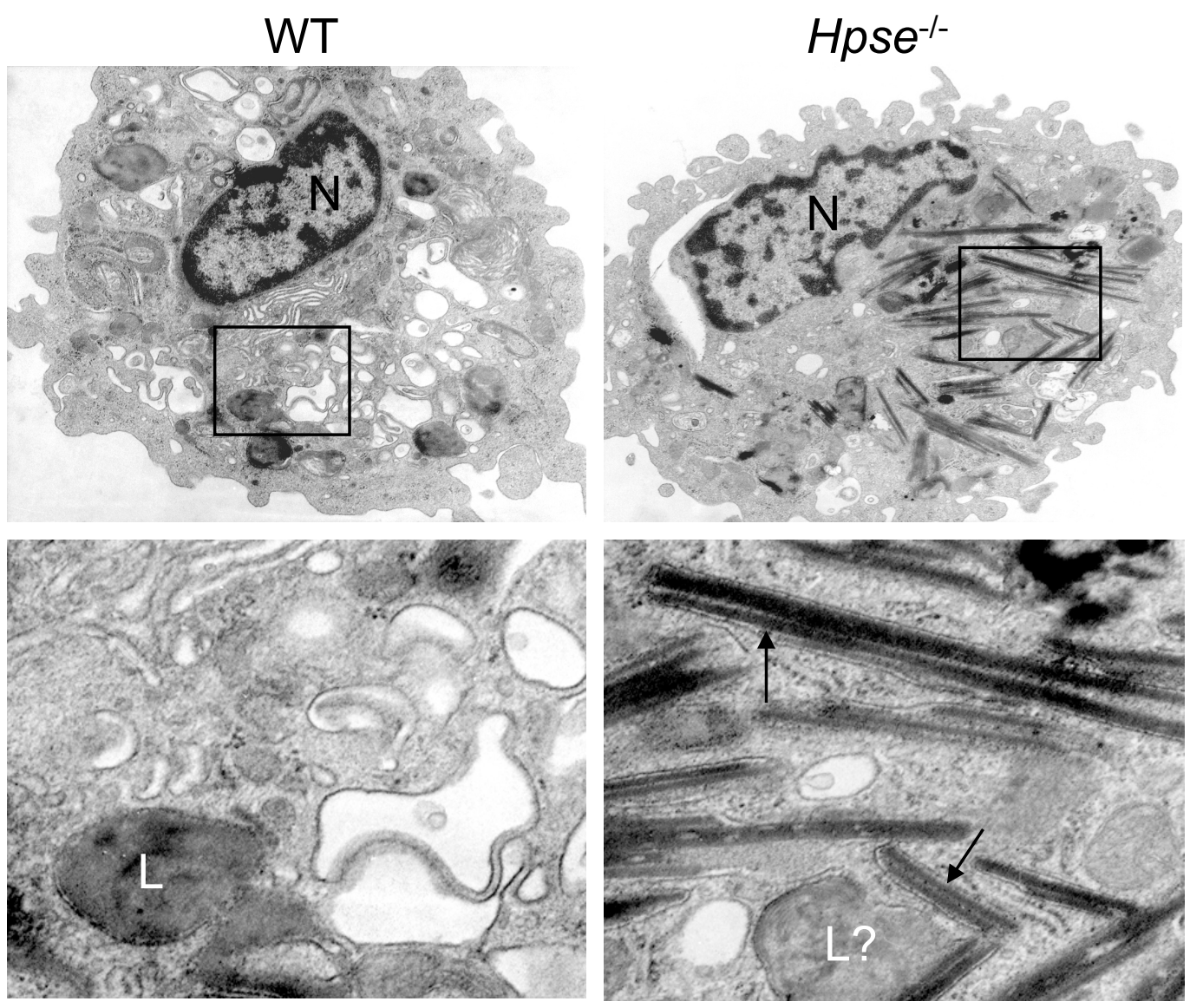
Fig.4

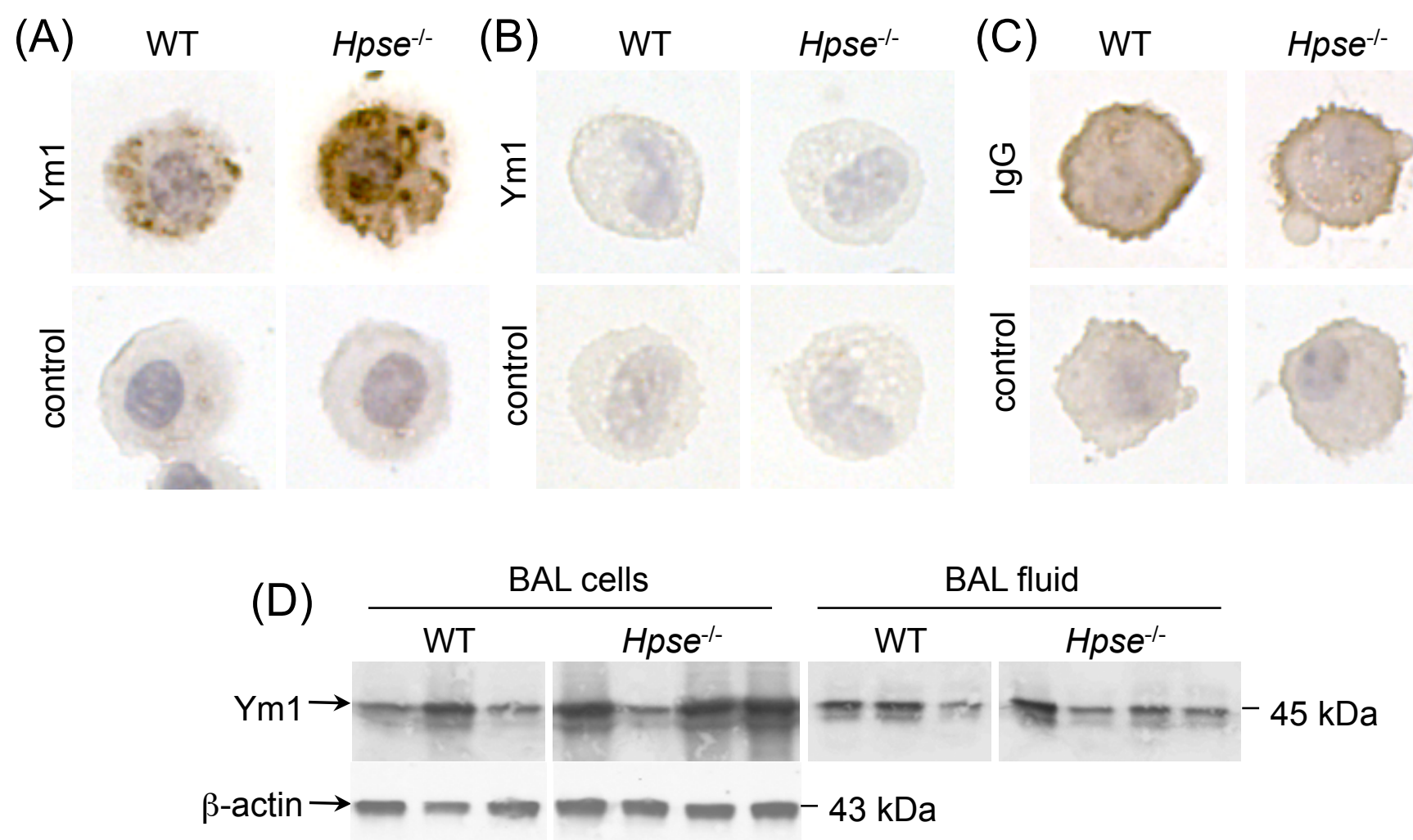

(E)

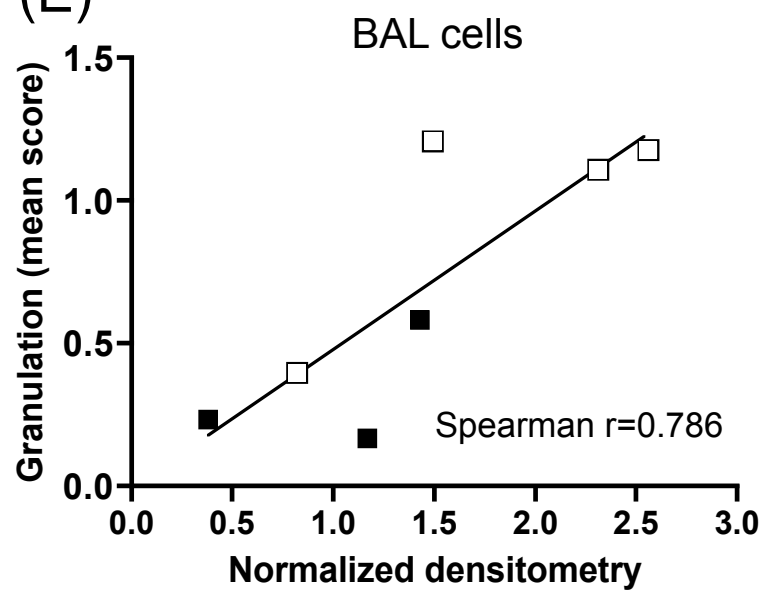

(F)

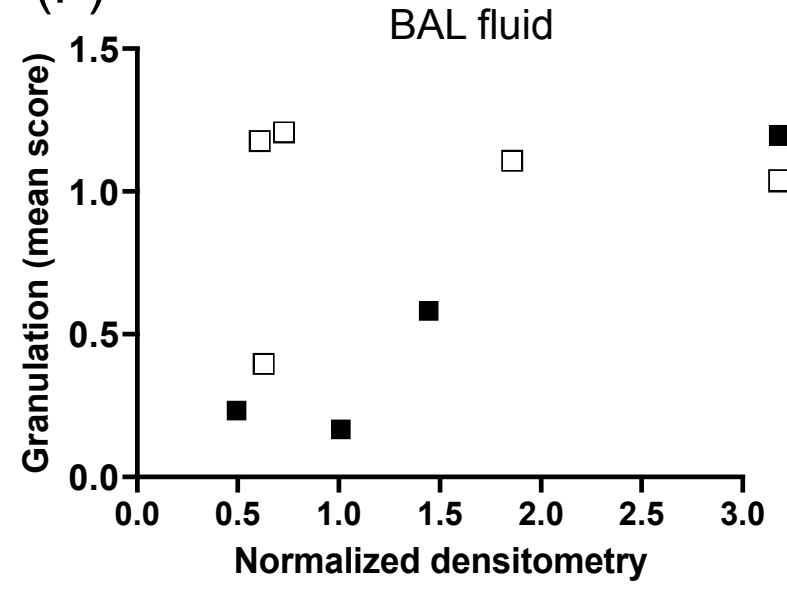


Fig.5

(A)
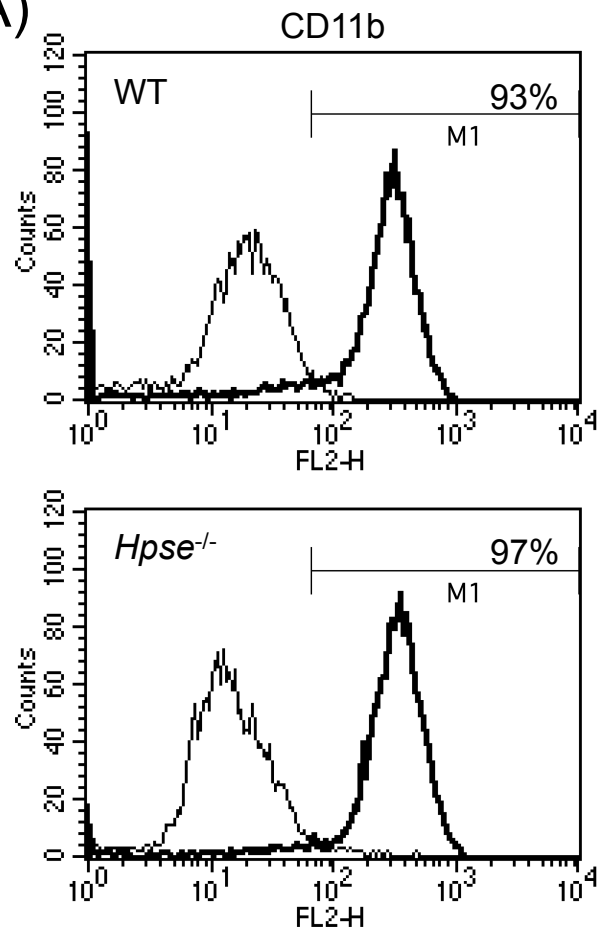

(B)

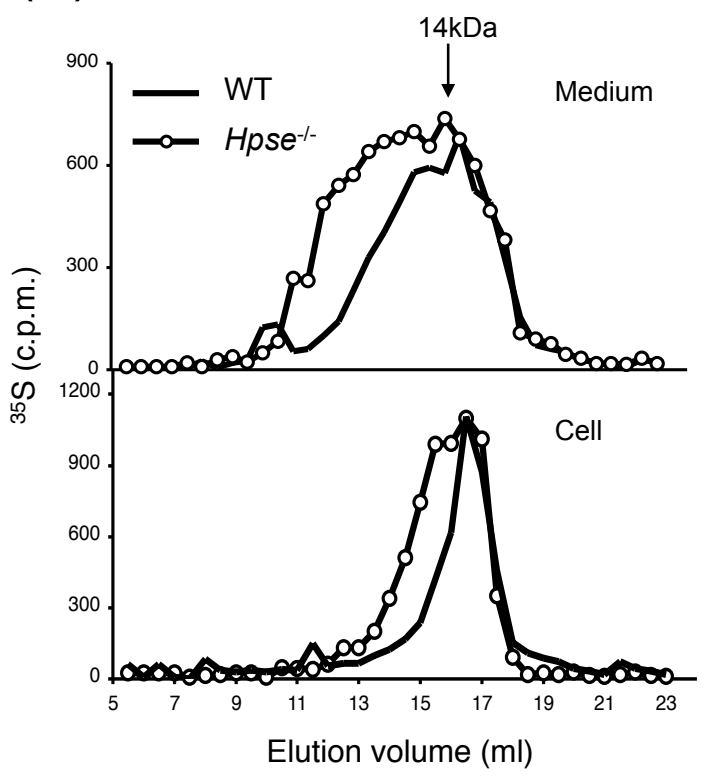

